\title{
RESPONSE TO INSULIN AS AN INDEX TO THE DIETARY MANAGEMENT OF DIABETES
}

\author{
By CYRIL M. MAcBRYDE \\ (From the Department of Medicine, Washington University School of Medicine, the Barnes \\ Hospital, and the Washington University Clinics, St. Louis)
}

(Received for publication May 20, 1936)

Recently there has developed a widespread trend toward allowing relatively large amounts of carbohydrate in the diabetic diet. It should be emphasized, however, that the use of higher carbohydrate diets is not new; Donkin's milk treatment (1874) may have provided 250 grams of carbohydrate daily, and the "cures" of von Düring, Mosse, von Noorden and others match in carbohydrate content many of those advised today (1). Despite the good results obtained in some cases with such diets, the treatment of diabetes was in general carried out upon principles established by Naunyn (2), who found it usually necessary to limit carbohydrate to very small amounts. It was still the common opinion of students of the disease that deleterious results followed the excessive consumption of carbohydrate, with loss of sugar tolerance and deterioration in the clinical condition of the patient.

Strict limitation of the carbohydrate in the diet has in general proved quite satisfactory treatment, and its use is based upon long clinical experience and is supported by excellent laboratory evidence. The lowest carbohydrate diets, introduced by $F$. M. Allen et al. (3), were based upon the observation that partially depancreatized dogs lost tolerance with high carbohydrate intake, but gained tolerance with low carbohydrate. Patients under Allen's observation seemed to act similarly. The low caloric, low carbohydrate diets of the "Allen era" were followed for years quite generally and with considerable success. It was the common opinion that excessive carbohydrate ingestion might exhaust the diabetic's pancreas.

More recent efforts to find the optimum diabetic diet have led in widely divergent directions. Newburgh and Marsh (4) and Petrén (5) found that high fat was well tolerated by many patients, and advised keeping the carbohydrate very low. Rosenberg (6) reports good results with a similar regime. The introduction of insulin permitted the use of higher carbohydrate and encouraged many at least temporarily to forget the principle of "sparing" the carbohydrate metabolism. The leaders in the recent movement toward higher carbohydrate diets (Sansum; Adlersberg and Porges; Geyelin; and Rabinowitch (7)) have demonstrated that many diabetics experience a definite improvement in health and may gain in sugar tolerance upon diets higher in carbohydrate than previously thought advisable. This has been a significant advance in our knowledge, reemphasizing the observations of earlier workers and tending to modify previously accepted conceptions of the pathological physiology of the disease. It has been observed that many patients do not gain tolerance with high carbohydrate, but the fact that many do requires a revision of the idea that "pancreas-sparing" is necessary in the treatment of all patients with diabetes.

It is quite generally accepted that as concerns total calories, normal or slight undernutrition is advisable, and that 0.75 to 1.25 grams protein per kilogram per day should be given. Concerning the carbohydrate-fat ratio, however, on one side we find advocates of high fat to rest the pancreas, and on the other those recommending high carbohydrate to stimulate the pancreas. That this is true now, twelve years after the introduction of insulin, emphasizes that the use of the pancreatic hormone has so far not helped much in solving the problem. With so many theoretical, practical and experimental arguments on each side, it is not surprising to find the conservative, moderate fat, moderate carbohydrate regime as advocated by Joslin (8) so widely used, since it represents a compromise between the two extremes.

It seemed to us, however, an important observation that some of our diabetic patients gained tolerance with high carbohydrate, while others showed no tendency to gain, or even lost tolerance. It seemed possible that there might be some fundamental difference in the type of diabetes in pa- 
tients who responded so differently. The widespread use of the conservative, compromise type of diet might conceal latent possibilities of great improvement in glucose tolerance, or might in some cases even cause loss of tolerance.

Accordingly an investigation was undertaken to determine whether diabetics who will gain tolerance on high carbohydrate can be clinically distinguished from those who will not. The primary object was to find, if possible, a method for deciding upon the optimum type of diet for each individual diabetic. The optimum diet should supply the necessary calories in palatable form with the lowest possible insulin dosage, and should permit the development of the greatest possible carbohydrate tolerance.

\section{RELATIVE INSULIN RESPONSE}

Three years ago studies were reported (9) confirming the observations of Falta and Boller (10) upon the frequency of relative insulin-resistance in diabetes. Since then we have found that the diabetics we have studied have tended to fall into two groups, the relatively insulin-sensitive, and the relatively insulin-resistant. The insulinresistance here described is not that commonly indicated by the term, in which several hundred units per day are required. Infection, coma, hyperthyroidism, pituitary and adrenal disorders, hepatic and various cutaneous diseases account in most instances for temporary marked unresponsiveness to insulin. The resistant patients in this study showed a relative lack of response to insulin slight to moderate in degree and not explainable upon the basis of any discernible complications. The relative degree of insulin sensitivity tended to remain as a persistent characteristic of the individual.

Studies were planned to determine whether the two groups differed in their response to high carbohydrate diets. Fifteen patients were chosen, each of them being intensively studied for three weeks to three months in the hospital, and from three months to three or more years in the outpatient department. The only criterion employed in the selection of cases was the individual's willingness to cooperate. Observations have been made upon the response of these patients to high and low carbohydrate diets, and the influence of these diets upon the concentration of blood sugar, the glycosuria, the ketonuria, the insulin requirement, the glucose tolerance and relative insulin sensitivity.

\section{METHODS OF STUDY}

The studies are best described under three headings: (1) determinations of relative insulin-sensitivity; (2) hospital studies; (3) observations in the outpatient department.

\section{Relative response to insulin}

(a) Blood sugar curves for four hours following the subcutaneous injection of one unit of insulin per 10 pounds body weight into the fasting patient were determined.

(b) Intravenous insulin tolerance was determined as a check upon the subcutaneous method. One-fifteenth unit per kilogram body weight was given intravenously to the fasting patient, blood samples for sugar analysis being collected every fifteen minutes for one and one-half hours.

(c) Tolerated overdose. After the insulin requirement upon the standard diet was determined, the total dose of insulin was gradually increased from day to day until the patient experienced a hypoglycemic reaction. The tolerated overdose may be defined as the amount of insulin above the insulin requirement which a patient will tolerate per day without having a reaction.

(d) Glucose equivalent. The glucose equivalent, representing the number of grams of glucose metabolized per unit of insulin, was calculated by dividing the number of grams excreted by the number of insulin units required to eliminate the glycosuria.

Laboratory methods :

(a) Blood sugar determinations were performed on capillary blood by the method of Somogyi (11). These are "true sugar" readings and average 15 to $20 \mathrm{mgm}$. per 100 cc. lower than those obtained by the older methods. Ante- and post-cibos blood sugars (fasting, and $21 \frac{1}{2}$ hours after breakfast) served as frequent checks upon changes in tolerance.

(b) The twenty-four hour urines were tested daily for glucose and acetone bodies. The quantitative excretion was determined by the Shaffer-Hartmann method (12).

(c) Glucose tolerance tests were performed by estimation of the fasting blood sugar, and at one-half, one, two and three hours after the ingestion of 0.8 gram dextrose per pound of body weight. For comparison with the figures of others the relative rise in the blood sugar is indicated by the percentage increase above the fasting figure. A more accurate measurement expresses the differences in area of the curves in "milligram-minutes" (Table IV). The figures given represent the rise above the fasting level in each case, a rise of 20 milligrams lasting for one hour, for example, being expressed as a rise of 1200 milligram-minutes.

(d) The total glucose value of the diets was calculated as 10 per cent of the fat plus 58 per cent of the protein plus 100 per cent of the carbohydrate. 
Accurate dietary regulation and prompt collection and preservation of specimens for analysis was carried out by the trained staff of the Tirrill Metabolism Ward.

\section{Studies on patients in the hospital}

(a) Control diets were given supplying 27.3 calories per kilogram body weight. This was considered a maintenance energy value for patients spending most of their time in bed, but permitted to be up and about in the ward. One gram of protein, 1.7 grams of fat and 2 grams of carbohydrate per kilogram per day were chosen as representing an average between the high fat and high carbohydrate types of diet. The protein supplied 15 per cent, the fat 56 per cent and the carbohydrate 29 per cent of the total caloric value. In the high carbohydrate "normal type" diet used by Geyelin (7) fat furnishes 33 per cent of the calories or less, and the carbohydrate 53 per cent or over. Rabinowitch's high carbohydrate diets (7) supplied 22 per cent of the calories from fat, 63 per cent from carbohydrate. In the Newburgh high fat diets (4) fat formed 75 per cent, carbohydrate 10 per cent, of the caloric intake.

The daily glycosuria in grams was measured, and the daily amount of insulin necessary to keep the blood sugar within approximately normal limits, and the glycosuria below 10 grams per 24 hours was determined. No effort was made to get the urine absolutely sugar-free, since we should then have been unable to tell whether the actual daily insulin requirement was being exceeded.

After a period upon the control regimen sufficiently long to avoid marked variations in daily blood sugar or daily glycosuria, the effect of alterations in the diet was studied. These were arranged in such a way as to involve no change in the protein or total caloric intake or in the insulin dosage. Test diets of the high fat type and of the high carbohydrate type were given, and the effects upon glycosuria and glycemia noted.

In some of the cases studied, glucose tolerance and insulin tolerance tests, while on the control regime and after the high carbohydrate period, were performed.

In certain instances the addition of fat or carbohydrate to the control diet was employed to determine the effect of adding calories above the control level in the form of carbohydrate or fat.

(b) In some cases a second type of control diet was used supplying 60 grams protein, 125 grams fat, and 150 grams carbohydrate, 1965 calories, without regard to the patient's weight. The effect of added carbohydrate and fat could then be studied without regard to certain theoretical objections to the first method. In thin patients receiving only 1500 or 1600 calories on the first type of control diet, for example, changes in tolerance might be ascribed to undernutrition, while obese patients receiving 2700 calories would certainly be subject to possible tolerance changes from excessive food intake as well as to possible influences of the high carbohydrate or high fat diets.

\section{Outpatient observations}

The effects of varying the proportions of fat and carbohydrate in the diet of patients measuring and pre- paring their own food at home and seen at intervals of a week or more could not of course be as closely judged as those in the hospital. Certain facts, however, make these observations of considerable importance, and permit placing them on a plane of equal significance with the hospital studies.

All of the outpatients had previously been in the hospital where the technique of weighing and measuring diets and of testing the urine for sugar had been well learned. Each outpatient had also been a subject of one of the hospital studies, so that hospital and outpatient studies serve as checks on each other. Frequent urine tests at home, at least once daily, were recorded, and the record brought with the patient on each visit. Frequent blood sugar determinations were made. On each visit an analysis of the patient's dietary control was made by the dietitian. Adherence to the prescribed total caloric intake could be checked by the frequent records of body weight.

With these considerations in mind, plus the fact that changes in carbohydrate tolerance observed over long periods of time should naturally be more significant in the study of diabetes, the results seem particularly significant.

\section{RESULTS}

\section{Relative response to insulin}

(a) Subcutaneous insulin tolerance. The patients fell into two groups, the relatively insulin sensitive and the relatively insulin resistant (Table I). The sensitive group of eight patients exhibited a marked fall in the blood sugar, ranging

TABLE I

Insulin tolerance (1.0 unit per 10 pounds subcutaneously)

\begin{tabular}{c|c|c|c|c|c|c}
\hline $\begin{array}{c}\text { Case } \\
\text { num- } \\
\text { ber }\end{array}$ & \multicolumn{5}{c|}{ Blood sugar (mgm. per 100 cc.) } & $\begin{array}{c}\text { Per } \\
\text { cent } \\
\text { fall }\end{array}$ \\
\cline { 2 - 7 } & Fasting & 1 hour & 2 hours & 3 hours & 4 hours & \\
\hline \multicolumn{6}{c}{ INSULIN-SENSITIVE TYPE } \\
\hline 1 & 156 & 124 & 71 & $41(\mathrm{r}) *$ & 41 & 74 \\
2 & 234 & 179 & 143 & 66 & 58 & 75 \\
3 & 176 & 134 & $39(\mathrm{r}) *$ & 52 & 70 & 70 \\
4 & 119 & 88 & 46 & 45 & 45 & 62 \\
5 & 218 & 133 & 87 & 59 & 63 & 73 \\
6 & 212 & 147 & 111 & 61 & 71 & 71 \\
7 & 142 & 119 & 100 & 42 & 48 & 70 \\
8 & 202 & 175 & 123 & 68 & 81 & 61 \\
\hline
\end{tabular}

INSULIN-RESISTANT TYPE

\begin{tabular}{r|r|r|r|r|r|r}
\hline 9 & 164 & 132 & 111 & 125 & 128 & 32 \\
10 & 134 & 137 & 119 & 110 & 80 & 40 \\
11 & 191 & 166 & 128 & 91 & 102 & 53 \\
12 & 119 & 96 & 99 & 71 & 82 & 40 \\
13 & 115 & 96 & 80 & 80 & 76 & 34 \\
14 & 212 & 206 & 155 & 100 & 110 & 53 \\
15 & 173 & 155 & 115 & 102 & 111 & 40 \\
\hline
\end{tabular}

* $(r)$ indicates symptoms of hypoglycemia. 
from 61 to 75 per cent of the fasting levels. All of them reached a blood sugar level of 68 or below, the average of the lowest determinations being $52 \mathrm{mgm}$. per cent. Definite signs of hypoglycemia were observed in every patient of this group and two of them experienced quite severe reactions.

The relatively resistant patients showed much less response. The per cent fall ranged from 32 to 53 ; none of them reached a point below 71 mgm. per $100 \mathrm{cc}$., and the average of the lowest determinations was $90 \mathrm{mgm}$. Signs of hypoglycemia were minimal or absent.

(b) Intravenous insulin tolerance tests upon three of the patients in each group indicated that possible differences in absorption of the subcutaneous insulin could not explain the observed differences in response. In each instance a patient sensitive to subcutaneous insulin was found also to be sensitive to intravenous insulin. Those relatively resistant showed relatively poor response to both the subcutaneous and the intravenous hormone (Table II). The relative fall in per cent ranged from 34 to 45 in the sensitive group, and from 12 to 22 in the resistant type.

TABLE II

Insulin tolerance (1/15 unit per kilogram body weight intravenously)

\begin{tabular}{|c|c|c|c|c|c|c|c|c|}
\hline \multirow{2}{*}{$\begin{array}{c}\text { Case } \\
\text { num- } \\
\text { ber }\end{array}$} & \multicolumn{7}{|c|}{ Blood sugar (mgm. per $100 \mathrm{cc}$.) } & \multirow{2}{*}{$\begin{array}{l}\text { Per } \\
\text { cen } \\
\text { fall }\end{array}$} \\
\hline & $\underset{\text { ing }}{\text { Fast- }}$ & $\begin{array}{c}15 \\
\text { min- } \\
\text { utes }\end{array}$ & $\underset{\text { min- }}{30}$ & $\begin{array}{c}45 \\
\min - \\
\text { utes }\end{array}$ & $\underset{\substack{60 \\
\text { min- }}}{\text { utes }}$ & $\begin{array}{c}75 \\
\text { min- } \\
\text { utes }\end{array}$ & $\underset{\text { min- }}{90}$ & \\
\hline \multicolumn{9}{|c|}{ INSULIN-SENSITIVE TYPE } \\
\hline $\begin{array}{l}1 \\
3 \\
8\end{array}$ & $\begin{array}{l}213 \\
156 \\
189\end{array}$ & $\begin{array}{l}200 \\
127 \\
170\end{array}$ & $\begin{array}{l}166 \\
110 \\
156\end{array}$ & $\begin{array}{r}159 \\
96 \\
145\end{array}$ & $\begin{array}{r}141 \\
86 \\
130\end{array}$ & $\begin{array}{r}145 \\
94 \\
112\end{array}$ & $\begin{array}{r}160 \\
98 \\
135\end{array}$ & $\begin{array}{l}34 \\
45 \\
40\end{array}$ \\
\hline \multicolumn{9}{|c|}{ INSULIN-RESISTANT TYPE } \\
\hline $\begin{array}{l}11 \\
14 \\
15\end{array}$ & $\begin{array}{l}182 \\
206 \\
194\end{array}$ & $\begin{array}{l}177 \\
202 \\
190\end{array}$ & $\begin{array}{l}175 \\
199 \\
173\end{array}$ & $\begin{array}{l}174 \\
181 \\
157\end{array}$ & $\begin{array}{l}170 \\
182 \\
163\end{array}$ & $\begin{array}{l}168 \\
192 \\
151\end{array}$ & $\begin{array}{l}158 \\
194 \\
160\end{array}$ & $\begin{array}{l}13 \\
12 \\
22\end{array}$ \\
\hline
\end{tabular}

(c) The tolerated overdose in those patients found to be sensitive in the insulin tolerance tests was in every case practically zero. An increase of as much as three or five units produced hypoglycemia, while a reduction of three or five units below the required amount led promptly to glycosuria and hyperglycemia (Table III).
TABLE III

Clinical characteristics and insulin requirements

\begin{tabular}{|c|c|c|c|c|c|c|c|}
\hline $\begin{array}{c}\text { Case } \\
\text { num- } \\
\text { ber }\end{array}$ & Age & Sex & $\underset{\text { tion }}{\text { Nutri- }}$ & $\begin{array}{l}\text { Blood } \\
\text { press- } \\
\text { ure }\end{array}$ & $\begin{array}{c}\text { Insulin } \\
\text { require- } \\
\text { ment }\end{array}$ & $\begin{array}{l}\text { Toler- } \\
\text { ated } \\
\text { over- } \\
\text { dose }\end{array}$ & $\begin{array}{c}\text { Glucose } \\
\text { equivalent }\end{array}$ \\
\hline & years & & & $\underset{H \boldsymbol{g}}{\operatorname{mm}}$ & $\begin{array}{c}\text { units } \\
\text { per } \\
\text { \&4 } \\
\text { hours }\end{array}$ & $\begin{array}{c}\text { units } \\
\text { per } \\
\text { \&4 } \\
\text { hours }\end{array}$ & \\
\hline
\end{tabular}

INSULIN-SENSITIVE

\begin{tabular}{|c|c|c|c|c|c|c|c|}
\hline $\begin{array}{l}1 \\
2 \\
3\end{array}$ & $\begin{array}{l}31 \\
46 \\
47\end{array}$ & $\begin{array}{l}\mathbf{M} \\
\mathbf{M} \\
\mathbf{F}\end{array}$ & $\begin{array}{l}\text { Thin } \\
\text { Thin } \\
\text { Slightly }\end{array}$ & $\begin{array}{l}104 / 70 \\
106 / 74 \\
138 / 74\end{array}$ & $\begin{array}{r}30 \\
75 \\
0\end{array}$ & $\begin{array}{l}\mathbf{0} \\
\mathbf{0}\end{array}$ & $\begin{array}{l}1.3,2.5,2.1 \\
1.9,2.3 \\
3.2\end{array}$ \\
\hline $\begin{array}{l}4 \\
5 \\
6 \\
7 \\
8\end{array}$ & $\begin{array}{l}29 \\
38 \\
44 \\
53 \\
33\end{array}$ & $\begin{array}{l}\mathbf{M} \\
\mathbf{F} \\
\mathbf{F} \\
\mathbf{F} \\
\mathbf{M}\end{array}$ & $\begin{array}{l}\text { Normal } \\
\text { Normal } \\
\text { Thin } \\
\text { Obese } \\
\text { Thin }\end{array}$ & $\begin{array}{l}110 / 70 \\
105 / 70 \\
105 / 70 \\
174 / 88 \\
120 / 70\end{array}$ & $\begin{array}{r}50 \\
50 \\
38 \\
0 \\
70\end{array}$ & $\begin{array}{l}\mathbf{0} \\
\mathbf{0} \\
\mathbf{0}\end{array}$ & $\begin{array}{l}1.1 \\
1.8 \\
2.9 \\
1.2\end{array}$ \\
\hline
\end{tabular}

INSULIN-RESISTANT

\begin{tabular}{|c|c|c|c|c|c|c|c|}
\hline $\begin{array}{r}9 \\
10 \\
11\end{array}$ & $\begin{array}{l}56 \\
68 \\
43\end{array}$ & $\begin{array}{l}\mathbf{M} \\
\mathbf{F}\end{array}$ & $\begin{array}{l}\text { Normal } \\
\text { Normal } \\
\text { Very }\end{array}$ & $\begin{array}{l}135 / 70 \\
140 / 80 \\
190 / 120\end{array}$ & $\begin{array}{l}25 \\
60 \\
50\end{array}$ & $\begin{array}{l}30 \\
75 \\
60\end{array}$ & $\begin{array}{l}0.80,0.90 \\
0.22,0.33 \\
0.37,0.35,0.40\end{array}$ \\
\hline 12 & 53 & $\mathbf{M}$ & Very & $140 / 80$ & 40 & 35 & 1.1 \\
\hline $\begin{array}{l}13 \\
14 \\
15\end{array}$ & $\begin{array}{l}33 \\
53 \\
55\end{array}$ & $\begin{array}{l}\mathbf{F} \\
\mathbf{F}\end{array}$ & $\begin{array}{l}\text { Obese } \\
\text { Obese } \\
\text { Obese }\end{array}$ & $\begin{array}{l}130 / 85 \\
135 / 70 \\
135 / 80\end{array}$ & $\begin{array}{l}45 \\
55 \\
70\end{array}$ & $\begin{array}{l}60 \\
65 \\
15\end{array}$ & $\begin{array}{l}0.56,0.50 \\
0.70,0.77 \\
0.30\end{array}$ \\
\hline
\end{tabular}

In the resistant group, however, increase in the insulin dosage had a relatively slight effect. In the seven patients in this group (Cases 9 through 15) the tolerated daily overdose ranged from 15 to 75 units. In Case 9 the requirement was 25 units, the tolerated overdose 30 units, so that 55 units daily were required to provoke hypoglycemia. In Case 10, the requirement was 60 , the tolerated overdose 75 , a total of 135 units causing the first evidences of insulin excess. In Cases 11,13 and 14 the required dose could also be more than doubled without producing symptoms of hypoglycemia.

(d) The glucose equivalents, representing the number of grams of glucose metabolized per unit of insulin, would be expected to be higher in the insulin-sensitive group. Such was found to be the case, the values in this group ranging from 1.1 to 3.2 and averaging over 2 grams (Table III). In the resistant type the values averaged 0.55 gram, ranging from 0.22 to 1.1 . On the average, then, by this criterion, there appears to be approximately four times as much effect per unit in the sensitive group as in the resistant group. 


\section{Clinical characteristics}

While it is our impression that the clinical features in the two groups differ significantly (9), there is considerable overlapping of clinical characteristics (Table III). Those patients showing relative resistance are usually older, frequently are obese, and often have vascular hypertension. There is little tendency to acidosis, while the sensitive group develop acidosis and coma much more easily. The sensitive group are usually younger, are often thin, and have as a rule low blood pressures. It is doubtful, however, whether clinical features alone will serve to distinguish the two types. They cannot be distinguished as to severity, since the insulin requirement is on the average higher in the resistant group, but acidosis occurs more frequently in the sensitive type.

TABLE IV

Effect of high carbohydrate diets upon insulin tolerance and glucose tolerance

\begin{tabular}{|c|c|c|c|c|c|}
\hline \multirow[t]{2}{*}{$\begin{array}{c}\text { Case } \\
\text { number* }\end{array}$} & \multirow{2}{*}{$\begin{array}{c}\begin{array}{c}\text { Diet } \\
(27.3 \\
\text { calories } \\
\text { per kgm.) }\end{array} \\
\begin{array}{c}\text { grams carbo- } \\
\text { hydrate per } \\
\text { kilogram }\end{array}\end{array}$} & \multirow{2}{*}{$\begin{array}{c}\begin{array}{c}\text { Subcu- } \\
\text { taneous } \\
\text { insulin } \\
\text { tolerance }\end{array} \\
\begin{array}{c}\text { per cent } \\
\text { fall }\end{array}\end{array}$} & \multirow{2}{*}{$\begin{array}{c}\begin{array}{c}\text { Intra- } \\
\text { venous } \\
\text { insulin } \\
\text { tolerance }\end{array} \\
\begin{array}{c}\text { per cent } \\
\text { fall }\end{array}\end{array}$} & \multicolumn{2}{|c|}{$\begin{array}{c}\text { Glucose } \\
\text { tolerance }\end{array}$} \\
\hline & & & & 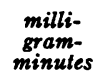 & $\begin{array}{c}\text { per cent } \\
\text { rise }\end{array}$ \\
\hline $1(\mathrm{~S})$ & 2 (6 days) & 74 & 34 & 21,705 & 67 \\
\hline $4(S)$ & $\begin{array}{l}3 \text { (7 days) } \\
2 \text { (6 days) }\end{array}$ & $\begin{array}{l}70 \\
62\end{array}$ & 32 & $\begin{array}{l}29,145 \\
23,220\end{array}$ & $\begin{array}{l}125 \\
251\end{array}$ \\
\hline & 3 (9 days) & 58 & & 37,995 & 280 \\
\hline $8(S)$ & 2 (11 days) & 61 & 40 & 18,600 & 103 \\
\hline $11(\mathrm{R})$ & 2 (7 days) & $\begin{array}{l}00 \\
53\end{array}$ & 38 & $\begin{array}{l}23,170 \\
13,560\end{array}$ & $\begin{array}{r}104 \\
92\end{array}$ \\
\hline & 3 (6 days) & 58 & & 12,120 & 68 \\
\hline $14(\mathrm{R})$ & 2 (16 days) & 53 & 12 & 37,380 & 122 \\
\hline & 3 (9 days) & 60 & 28 & 25,410 & 82 \\
\hline $15(\mathrm{R})$ & $\begin{array}{l}2 \text { (18 days) } \\
3 \text { (8 days) }\end{array}$ & $\begin{array}{l}40 \\
49\end{array}$ & $\begin{array}{l}22 \\
30\end{array}$ & $\begin{array}{r}24,270 \\
9,060\end{array}$ & $\begin{array}{l}96 \\
51\end{array}$ \\
\hline
\end{tabular}

* Cases 1, 4 and 8 (S) are relatively insulin-sensitive. Cases 11, 14 and $15(R)$ are relatively resistant to insulin.

The clinical characteristics, with the insulin requirements, tolerated overdoses, and glucose equivalents are summarized in Table III. Cases 1 through 8 are those who showed relative sensitivity in the insulin tolerance tests. Cases 9 through 15 were relatively resistant.

\section{Results of dietary studies}

In Tables IV, V and VI analyses are given of the responses to the various test diets. Of eight relatively insulin-sensitive patients studied in the hospital, seven showed no gain or a loss of tolerance following high carbohydrate diets; one sensi- tive patient gained tolerance. High fat, however, was relatively well borne and was followed in several instances by improved tolerance.

Seven relatively resistant patients studied in the hospital all showed definitely improved tolerance following high carbohydrate diets. Fat was as a rule poorly tolerated by this group and tended to impair tolerance.

In the outpatient studies over long periods of time five of six sensitive patients failed to gain tolerance with increased carbohydrate intake; one showed improved tolerance.

Five relatively resistant patients gained tolerance in a definite and remarkable manner upon progressively increasing the carbohydrate ingestion.

\section{The cause of improved carbohydrate tolerance resulting from high carbohydrate diets}

It is evident from these experiments that some patients with diabetes may be expected to gain tolerance when allowed large amounts of carbohydrate, while others either fail to gain or actually lose tolerance.

The early observation of Hamman and Hirschman (13) that the hyperglycemia resulting from the second of two equal ingested amounts of glucose by a normal subject is less than that following the first dose has been confirmed by Staub (14), Traugott (15), Foster (16), du Vigneaud and Karr (17), and Lennox (18). Lennox demonstrated that this was true whether the glucose be given orally or intravenously. This effect is now commonly referred to as the Staub-Traugott phenomenon.

Sweeney (19) investigating the effects of various diets upon normal subjects, and using the glucose tolerance test as an indicator, concluded that starvation or a high fat diet decreased the tolerance, protein diets had little effect, and high carbohydrate raised the tolerance. He explained this effect by supposing that the mechanism secreting insulin becomes increasingly sensitive to stimulation when frequently subjected to the higher blood sugar resulting from the high carbohydrate diets, while the absence of such stimulation caused insulin to be secreted less readily and in smaller amounts. 
TABLE V

Responses to various test diets

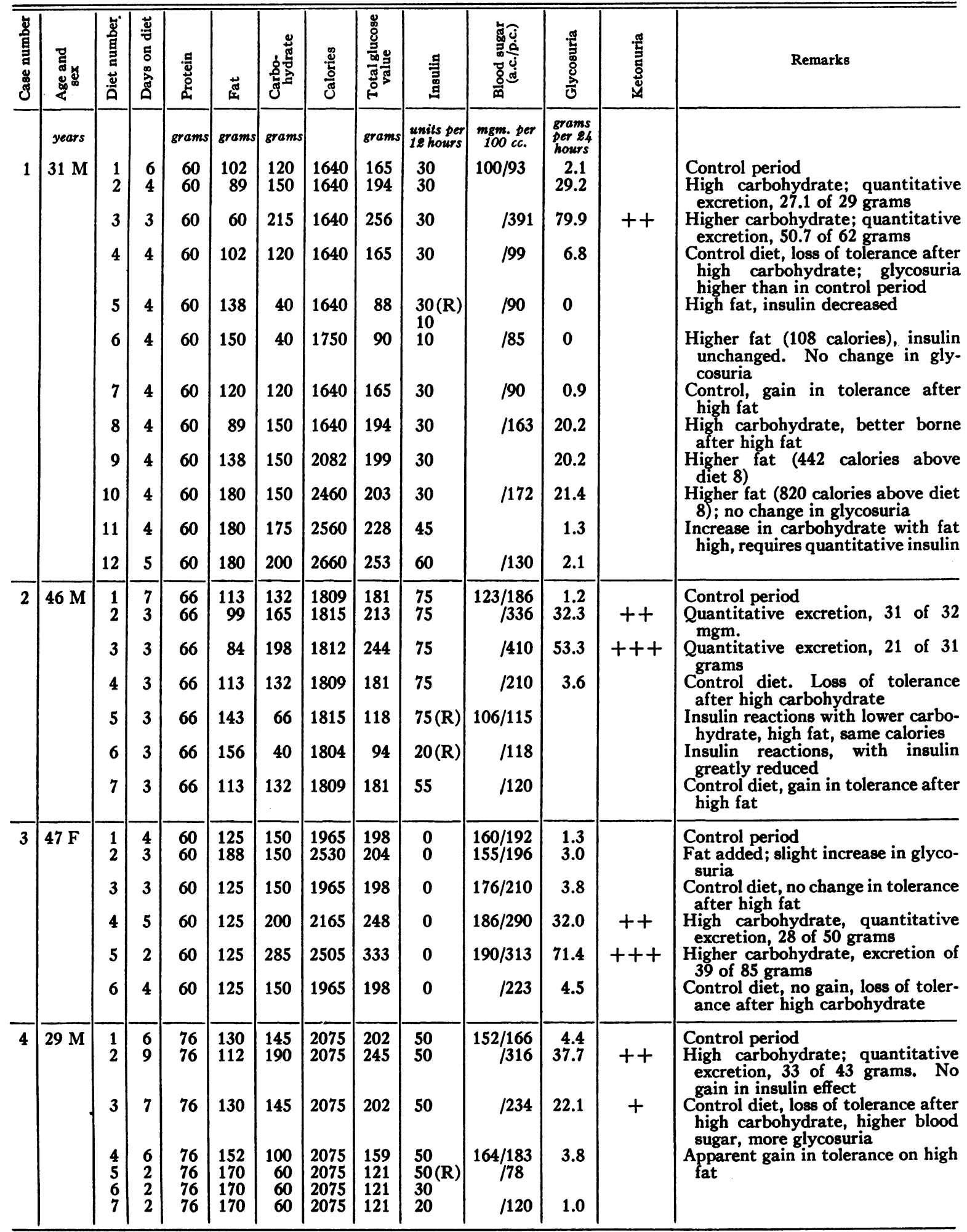


TABLE v-Continued

\begin{tabular}{|c|c|c|c|c|c|c|c|c|c|c|c|c|c|}
\hline 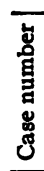 & 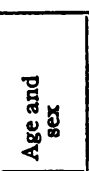 & 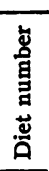 & 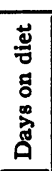 & 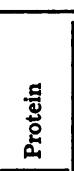 & 苛 & 蒡 & 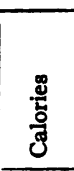 & 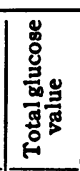 & 量 & 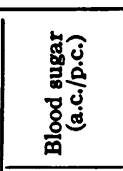 & 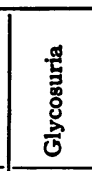 & 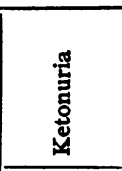 & Remarks \\
\hline \multirow{6}{*}{5} & years & & & grams & grams & grams & & grams & \begin{tabular}{|} 
units per \\
12 hours
\end{tabular} \mid & $\begin{array}{l}\text { mgm. per } \\
100 \text { cc. }\end{array}$ & $\begin{array}{l}\text { grams } \\
\text { per 24 } \\
\text { hours }\end{array}$ & \multirow{6}{*}{$\begin{array}{l}++ \\
++\end{array}$} & \multirow{6}{*}{$\begin{array}{l}\text { Low carbohydrate } \\
\text { Control diet } \\
\text { No loss of tolerance with high fat } \\
\text { added } \\
\text { Perhaps gain in tolerance after } \\
\text { high fat } \\
\text { Quantitative excretion, } 47 \text { of } 50 \\
\text { grams carbohydrate added } \\
\text { Excretion of } 21 \text { of } 25 \text { grams carbo- } \\
\text { hydrate added } \\
\text { No gain in tolerance after high car- } \\
\text { bohydrate }\end{array}$} \\
\hline & $38 \mathrm{~F}$ & $\begin{array}{l}1 \\
2 \\
3\end{array}$ & $\begin{array}{r}7 \\
13 \\
3\end{array}$ & $\begin{array}{l}60 \\
60 \\
60\end{array}$ & $\begin{array}{l}125 \\
125 \\
188\end{array}$ & $\begin{array}{r}80 \\
150 \\
150\end{array}$ & $\begin{array}{l}1685 \\
1965 \\
2530\end{array}$ & $\begin{array}{l}128 \\
198 \\
204\end{array}$ & $\begin{array}{l}40 \\
50 \\
50\end{array}$ & $\begin{array}{r}218 / \\
242 / 218 \\
/ 198\end{array}$ & $\begin{array}{l}1.6 \\
1.2 \\
1.1\end{array}$ & & \\
\hline & & 4 & 3 & 60 & 125 & 150 & 1965 & 198 & 50 & $/ 205$ & & & \\
\hline & & 5 & 3 & 60 & 125 & 200 & 2165 & 248 & 50 & $/ 242$ & 47.3 & & \\
\hline & & 6 & 3 & 60 & 125 & 235 & 2305 & 283 & 50 & $/ 414$ & 68.1 & & \\
\hline & & 7 & 3 & 60 & 125 & 150 & 1965 & 198 & 50 & $/ 230$ & 1.2 & & \\
\hline \multirow[t]{4}{*}{6} & $44 \mathrm{~F}$ & $\begin{array}{l}1 \\
2 \\
3\end{array}$ & $\begin{array}{r}14 \\
3 \\
3\end{array}$ & $\begin{array}{l}60 \\
60 \\
60\end{array}$ & $\begin{array}{l}125 \\
188 \\
125\end{array}$ & $\begin{array}{l}150 \\
150 \\
150\end{array}$ & $\begin{array}{l}1965 \\
2530 \\
1965\end{array}$ & $\begin{array}{l}198 \\
204 \\
198\end{array}$ & $\begin{array}{l}38 \\
38 \\
38\end{array}$ & $\begin{array}{l}/ 131 \\
/ 136 \\
/ 110\end{array}$ & $\begin{array}{l}14.0 \\
14.0 \\
10.7\end{array}$ & & \multirow{4}{*}{$\begin{array}{l}\text { Control period } \\
\text { No loss of tolerance with added fat } \\
\text { Control diet, gain in tolerance after } \\
\text { high fat, less glycosuria, blood } \\
\text { sugar lower } \\
\text { Increase of } 50 \text { grams carbohydrate, } \\
\text { glycosuria increased } 47 \text { grams } \\
\text { Increase of } 85 \text { grams carbohydrate, } \\
\text { glycosuria increase of } 55 \text { grams } \\
\text { Loss of tolerance after high carbo- } \\
\text { hydrate. Increased glycosuria } \\
\text { and higher blood sugar }\end{array}$} \\
\hline & & 4 & 3 & 60 & 125 & 200 & 2165 & 248 & 38 & $/ 246$ & 57.1 & ++ & \\
\hline & & 5 & 3 & 60 & 125 & 285 & 2505 & 333 & 38 & 1424 & 102.3 & ++++ & \\
\hline & & 6 & 3 & 60 & 125 & 150 & 1965 & 198 & 38 & $/ 212$ & 23.4 & & \\
\hline \multirow[t]{6}{*}{9} & $56 \mathrm{~F}$ & $\begin{array}{l}1 \\
2\end{array}$ & $\begin{array}{l}6 \\
4\end{array}$ & $\begin{array}{l}57 \\
57\end{array}$ & $\begin{array}{l}98 \\
67\end{array}$ & $\begin{array}{l}114 \\
171\end{array}$ & $\begin{array}{l}1518 \\
1518\end{array}$ & $\begin{array}{l}157 \\
211\end{array}$ & $\begin{array}{l}25 \\
25\end{array}$ & $\begin{array}{r}161 / 130 \\
/ 152\end{array}$ & $\begin{array}{l}5.7 \\
9.9\end{array}$ & & \multirow{6}{*}{$\begin{array}{l}\text { Control period } \\
\text { High carbohydrate, excretion of } 4.2 \\
\text { of } 54 \text { grams } \\
\text { Higher carbohydrate, excretion of } \\
5.8 \text { of } 71 \text { grams } \\
\text { Control diet, gain in tolerance after } \\
\text { high carbohydrate } \\
\text { High fat, low carbohydrate } \\
\text { Control diet, loss of tolerance fol } \\
\text { lowing high fat } \\
\text { Increase of } 171 \text { grams in carbohy } \\
\text { drate, increased excretion of only } \\
15 \text { grams } \\
\text { Insulin increase of } 25 \text { units. } 19.3 \\
\text { grams } \div 25=\text { glucose equivalent } \\
\text { of } 0.8\end{array}$} \\
\hline & & 3 & 4 & 57 & 35 & 245 & 1518 & 282 & 25 & /194 & 15.7 & & \\
\hline & & 4 & 4 & 57 & 98 & 114 & 1518 & 157 & 25 & $/ 101$ & 1.7 & & \\
\hline & & $\begin{array}{l}5 \\
6\end{array}$ & $\begin{array}{l}4 \\
4\end{array}$ & $\begin{array}{l}57 \\
57\end{array}$ & $\begin{array}{r}126 \\
98\end{array}$ & $\begin{array}{r}38 \\
114\end{array}$ & $\begin{array}{l}1518 \\
1518\end{array}$ & $\begin{array}{r}84 \\
157\end{array}$ & $\begin{array}{l}25 \\
25\end{array}$ & $\begin{array}{l}/ 93 \\
/ 146\end{array}$ & $\begin{array}{l}1.0 \\
7.5\end{array}$ & & \\
\hline & & 7 & 4 & 57 & 98 & 285 & 2250 & 328 & 25 & $/ 245$ & 22.5 & & \\
\hline & & 8 & 4 & 57 & 98 & 285 & 2250 & 328 & 50 & $/ 141$ & 3.2 & & \\
\hline \multirow[t]{5}{*}{10} & $68 \mathrm{M}$ & $\frac{1}{2}$ & $\begin{array}{l}7 \\
3\end{array}$ & $\begin{array}{l}60 \\
60\end{array}$ & $\begin{array}{r}102 \\
60\end{array}$ & $\begin{array}{l}120 \\
217\end{array}$ & $\begin{array}{l}1638 \\
1648\end{array}$ & $\begin{array}{l}165 \\
258\end{array}$ & $\begin{array}{l}60 \\
60\end{array}$ & $\begin{array}{r}134 / 161 \\
/ 184\end{array}$ & $\begin{array}{r}3.1 \\
13.1\end{array}$ & & \multirow{5}{*}{$\begin{array}{l}\text { Control period } \\
\text { High carbohydrate; increased ex } \\
\text { cretion of only } 10 \text { of } 93 \text { grams } \\
\text { Higher carbohydrate; increased ex } \\
\text { cretion of only } 4 \text { of } 43 \text { grams } \\
\text { Control diet, gain in tolerance } \\
\text { lower blood sugar, less glycosuria } \\
\text { High fat, blood sugar higher, more } \\
\text { glycosuria } \\
\text { Control diet, loss in tolerance after } \\
\text { high fat, higher blood sugar, more } \\
\text { glycosuria }\end{array}$} \\
\hline & & 3 & 3 & 60 & 40 & 262 & 1648 & 301 & 60 & /192 & 17.1 & & \\
\hline & & 4 & 3 & 60 & 102 & 120 & 1638 & 165 & 60 & $152 / 154$ & 0 & & \\
\hline & & 5 & 3 & 60 & 138 & 40 & 1642 & 89 & 60 & $/ 186$ & 1.0 & & \\
\hline & & 6 & 3 & 60 & 102 & 120 & 1638 & 165 & 60 & $/ 180$ & 3.5 & & \\
\hline \multirow[t]{3}{*}{11} & $43 \mathrm{~F}$ & $\begin{array}{l}1 \\
2 \\
3\end{array}$ & $\begin{array}{l}8 \\
4 \\
3\end{array}$ & $\begin{array}{l}60 \\
60 \\
60\end{array}$ & $\begin{array}{l}125 \\
188 \\
125\end{array}$ & $\begin{array}{l}150 \\
150 \\
150\end{array}$ & $\begin{array}{l}1965 \\
2530 \\
1965\end{array}$ & $\begin{array}{l}198 \\
204 \\
198\end{array}$ & $\begin{array}{l}50 \\
50 \\
50\end{array}$ & $\begin{array}{l}/ 138 \\
/ 141 \\
/ 191\end{array}$ & $\begin{array}{l}5.2 \\
6.4 \\
6.4\end{array}$ & & \multirow{3}{*}{$\begin{array}{l}\text { Control period } \\
\text { Added fat, increased glycosuria } \\
\text { Control diet, higher blood sugar } \\
\text { increased glycosuria after high fa } \\
\text { High carbohydrate; excretion of } \\
\text { only } 4.2 \text { of } 50 \text { grams } \\
\text { Higher carbohydrate, excretion of } \\
6.2 \text { of } 50 \text { grams }\end{array}$} \\
\hline & & 4 & 4 & 60 & 125 & 200 & 2165 & 248 & 50 & /158 & 10.6 & & \\
\hline & & 5 & 4 & 60 & 125 & 300 & 2565 & 348 & 50 & $/ 230$ & 16.8 & + & \\
\hline
\end{tabular}


TABLE v-Continued

\begin{tabular}{|c|c|c|c|c|c|c|c|c|c|c|c|c|c|}
\hline 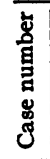 & 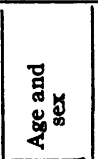 & 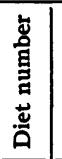 & 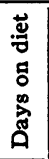 & 䞨 & 雚 & نู & हึँ & 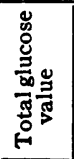 & 总 & 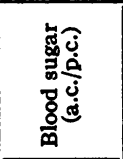 & 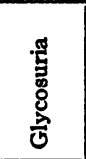 & 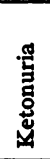 & Remarks \\
\hline 11 & $\begin{array}{l}\text { years } \\
43 \mathrm{~F}\end{array}$ & 6 & 4 & $\begin{array}{c}\text { grams } \\
60\end{array}$ & $\begin{array}{l}\text { grams } \\
125\end{array}$ & $\begin{array}{l}\text { grams } \\
150\end{array}$ & 1965 & $\begin{array}{c}\text { grams } \\
198\end{array}$ & $\begin{array}{l}\text { units per } \\
12 \text { hours } \\
50\end{array}$ & $\begin{array}{r}\text { mgm. per } \\
100 \text { cc. } \\
\quad / 110\end{array}$ & $\begin{array}{l}\text { grams } \\
\text { per } 84 \\
\text { hours } \\
0\end{array}$ & & $\begin{array}{l}\text { Control diet, no glycosuria, lower } \\
\text { blood sugar following high carbo- } \\
\text { hydrate }\end{array}$ \\
\hline 12 & $53 \mathrm{M}$ & $\begin{array}{l}1 \\
2 \\
3 \\
4 \\
5 \\
6\end{array}$ & $\begin{array}{l}6 \\
3 \\
3\end{array}$ & $\begin{array}{l}60 \\
60 \\
60 \\
60 \\
60 \\
60\end{array}$ & $\begin{array}{l}125 \\
188 \\
125\end{array}$ & $\begin{array}{l}200 \\
285 \\
150\end{array}$ & $\begin{array}{l}1965 \\
2530 \\
1965 \\
2165 \\
2505 \\
1965\end{array}$ & $\begin{array}{l}198 \\
204 \\
198 \\
248 \\
333 \\
198\end{array}$ & $\begin{array}{l}40 \\
40 \\
40 \\
40 \\
40 \\
40\end{array}$ & $\begin{array}{l}168 \\
/ 215 \\
/ 132 \\
/ 232 \\
/ 265 \\
/ 70\end{array}$ & $\begin{array}{r}2.6 \\
6.7 \\
1.8 \\
8.3 \\
19.2 \\
0\end{array}$ & & $\begin{array}{l}\text { Control period } \\
\text { Increased glycosuria, higher blood } \\
\text { sugar with high fat } \\
\text { Control diet, decreased tolerance } \\
\text { after high fat } \\
\text { High carbohydrate, excretion of } 6.5 \\
\text { of } 50 \text { added grams } \\
\text { Excretion of } 11 \text { of } 85 \text { added grams } \\
\text { Control diet, gain in tolerance fol- } \\
\text { lowing high carbohydrate }\end{array}$ \\
\hline 14 & $52 \mathrm{~F}$ & $\begin{array}{l}1 \\
2 \\
3 \\
4\end{array}$ & $\begin{array}{r}16 \\
5 \\
9\end{array}$ & $\begin{array}{l}100 \\
100 \\
100 \\
100\end{array}$ & $\begin{array}{l}170 \\
148\end{array}$ & $\begin{array}{l}200 \\
250 \\
300 \\
200\end{array}$ & $\begin{array}{l}2730 \\
2730 \\
2730\end{array}$ & $\begin{array}{l}265 \\
323 \\
371 \\
265\end{array}$ & $\begin{array}{l}55 \\
55 \\
55 \\
55\end{array}$ & $\begin{array}{r}206 / 110 \\
/ 151 \\
200 / 162 \\
/ 131 \\
/ 91\end{array}$ & $\begin{array}{l}3.4 \\
4.9 \\
6.6 \\
0\end{array}$ & & $\begin{array}{l}\text { Control period } \\
\text { High carbohydrate; small excretion } \\
\text { increase, } 1.5 \text { of } 58 \text { grams } \\
\text { Higher carbohydrate, increased ex- } \\
\text { cretion of only } 1.7 \text { of } 58 \text { grams } \\
\text { Control diet, improved tolerance } \\
\text { after high carbohydrate, lower } \\
\text { blood sugar, less glycosuria } \\
\text { Control diet, gain in tolerance per- } \\
\text { mits lower insulin dose }\end{array}$ \\
\hline 15 & $55 \mathrm{~F}$ & $\begin{array}{l}1 \\
2 \\
3\end{array}$ & $\begin{array}{r}18 \\
8 \\
7\end{array}$ & $\begin{array}{l}70 \\
70 \\
70\end{array}$ & $\begin{array}{r}119 \\
88 \\
119\end{array}$ & $\begin{array}{l}140 \\
210 \\
140\end{array}$ & $\begin{array}{l}1911 \\
1911 \\
1911\end{array}$ & $\begin{array}{l}193 \\
260 \\
193\end{array}$ & $\begin{array}{l}70 \\
70 \\
70\end{array}$ & $\begin{array}{r}175 / 166 \\
148 / 163 \\
/ 116\end{array}$ & $\begin{array}{l}4.9 \\
6.3 \\
0\end{array}$ & & $\begin{array}{l}\text { Control period } \\
\text { High carbohydrate, increased ex- } \\
\text { cretion of only } 1.4 \text { of } 67 \text { grams } \\
\text { Control diet, gain in tolerance, } \\
\text { lower blood sugar, less glycosuria }\end{array}$ \\
\hline
\end{tabular}

Macleod (20) offers a similar explanation of these phenomena, stating that a lower level of hyperglycemia acts as an adequate stimulus to insulin secretion after sensitization of the secreting apparatus by a previous rise in the blood sugar.

It seemed possible, however, as our experiments progressed, that the increased tolerance noted in some of our patients might be due to either of two factors: (1) increased secretion of insulin, or (2) increased sensitivity to endogenous insulin. Abderhalden and Wertheimer (21), and Bainbridge (22) showed that animals on high carbohydrate diets were much more sensitive to insulin than those which were receiving high fat. Hynd and Rotter (23) also noted that hypoglycemic convulsions were more easily produced in animals receiving large amounts of carbohydrate. Those patients who gained tolerance with high carbohydrate intake in this study fell in the relatively insulin-resistant group. It seemed possible that the increased tolerance was due to a better response to endogenous insulin. Insulin tolerance tests were therefore performed upon several resistant patients after periods upon the control diet containing 2 grams carbohydrate per kilogram body weight, and after high carbohydrate periods on 3 grams per kilogram. Glucose tolerance tests were performed before and after the high carbohydrate as a further check upon the effects of the diet, and because the only other similar curves upon diabetics we have been able to find were the rather inconclusive ones of Watson and Wharton (24). Similar insulin and glucose tolerance studies were made upon several of the sensitive group.

The results of these studies are shown in Table IV. Glucose tolerance curves in two of the three sensitive patients showed a higher percentage rise after the high carbohydrate diets, and in one there was practically no change. In all three resistant patients much lower curves were obtained on the high carbohydrate than on the control diets. In- 
sulin tolerance curves showed no change in sensitivity in the sensitive patients. A much more marked depression of the blood sugar was evident, however, in the relatively resistant group. This increased sensitivity to insulin was evident whether the insulin was given subcutaneously or intravenously.

While these results do not exclude the possibility of increased insulin production in the relatively resistant group, it seems probable that at least a part of the increased tolerance is due to the patient's greater sensitivity to his own insulin.

The sensitive group, on the contrary, seem rather constantly to respond maximally to endogenous or exogenous insulin, and their response is unchanged by diet. The glucose tolerance, however, is in some of the sensitive patients decreased upon high carbohydrate ingestion. It seems possible, therefore, that in these patients excessive strain upon the pancreatic islets has resulted in a diminished endogenous insulin supply. These patients may be thought of as having diabetes which is primarily pancreatic or insular, since in so many respects their reactions resemble those of Allen's depancreatized dogs.

In the insulin-resistant cases, on the other hand, evidence indicating pancreatic islet insufficiency is by no means so clear. The gain in tolerance upon high carbohydrate diets, accompanied by a definitely increased sensitivity to insulin, suggests that extra-pancreatic factors decreasing the effectiveness of endogenous insulin may be at least partly responsible for this type of diabetes.

\section{Extra-pancreatic factors in diabetes}

Our studies emphasize the fact that we can no longer consider diabetes a unitarian disease, caused solely by an inadequate production of insulin. Warren (25) and other pathologists have demonstrated that the non-diabetic pancreas may reveal changes previously described as the cause of diabetes, whereas the diabetic pancreas may in many instances show no definite disease. On the other hand, studies of recent years have shown that a number of other factors must be considered as exerting profound influences upon carbohydrate metabolism.

There is increasing evidence that the pituitary and adrenal glands may play an important part in the etiology of the common type of clinical diabetes (26). Hyperthyroidism is known to be

TABLE VI

Responses to various test diets

\begin{tabular}{|c|c|c|c|c|c|c|c|c|c|c|}
\hline 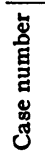 & 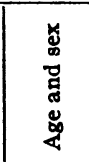 & 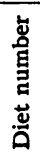 & 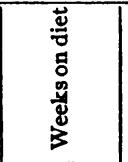 & 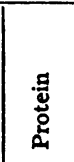 & 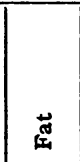 & 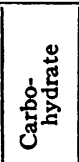 & $\begin{array}{l}\text { 韋 } \\
\text { 总 }\end{array}$ & 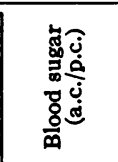 & $\frac{.05}{5}$ & Remarks \\
\hline 1 & $\begin{array}{c}\text { years } \\
31 \mathrm{M}\end{array}$ & $\begin{array}{l}2 \\
3 \\
4\end{array}$ & $\begin{array}{c}1 \text { year } \\
12 \\
4 \\
2\end{array}$ & $\begin{array}{c}\text { grams } \\
70 \\
60 \\
60 \\
60\end{array}$ & \begin{tabular}{|c|} 
grams \\
150 \\
180 \\
180 \\
180
\end{tabular} & $\begin{array}{r}\text { grams } \\
200 \\
200 \\
220 \\
200\end{array}$ & $\begin{array}{l}\text { units per } \\
\text { 24 hours } \\
75 \\
60 \\
65 \\
65\end{array}$ & $\begin{array}{r}\text { mgm. per } \\
100 \text { cc. } \\
/ 120 \\
/ 130 \\
/ 164\end{array}$ & $\begin{array}{c}++ \\
+\end{array}$ & $\begin{array}{l}\text { Fat raised in diet, insulin lowered, fat well } \\
\text { tolerated } \\
\text { Higher carbohydrate, requires increase in insulin } \\
\text { dosage, glycosuria and higher insulin require- } \\
\text { ment persist even when diet returned to pre- } \\
\text { vious level }\end{array}$ \\
\hline 3 & $47 \mathrm{~F}$ & $\begin{array}{l}1 \\
2 \\
3 \\
4 \\
5 \\
5\end{array}$ & $\begin{array}{r}8 \\
4 \\
32 \\
10 \\
2 \\
2\end{array}$ & $\begin{array}{l}60 \\
60 \\
60 \\
60 \\
60 \\
60\end{array}$ & $\begin{array}{r}125 \\
80 \\
125 \\
125 \\
110 \\
100\end{array}$ & $\begin{array}{r}100 \\
70 \\
150 \\
70 \\
120 \\
100\end{array}$ & $\begin{array}{l}\mathbf{0} \\
\mathbf{0} \\
\mathbf{0} \\
\mathbf{0} \\
\mathbf{0} \\
\mathbf{0}\end{array}$ & $\begin{array}{l}/ 180 \\
/ 112 \\
/ 196 \\
/ 168 \\
/ 170\end{array}$ & $\begin{array}{l}++ \\
++ \\
++ \\
+\end{array}$ & $\begin{array}{l}\text { Carbohydrate decreased because of glycosuria } \\
\text { Trial of higher carbohydrate } \\
\text { Carbohydrate decreased because of glycosuria } \\
\text { Trial of higher carbohydrate } \\
\text { No gain in tolerance }\end{array}$ \\
\hline 5 & $38 \mathrm{~F}$ & $\begin{array}{l}1 \\
2 \\
3 \\
4 \\
5 \\
6\end{array}$ & $\left|\begin{array}{c}10 \text { years } \\
10 \\
4 \\
2 \\
1 \\
3\end{array}\right|$ & $\begin{array}{l}60 \\
60 \\
50 \\
60 \\
60 \\
60\end{array}$ & $\begin{array}{r}185 \\
100 \\
50 \\
125 \\
125 \\
125\end{array}$ & $\begin{array}{r}50 \\
80 \\
100 \\
150 \\
200 \\
150\end{array}$ & $\begin{array}{l}10 \\
20 \\
25 \\
30 \\
40 \\
30\end{array}$ & $\begin{array}{r}173 / \\
242 / 218 \\
/ 260 \\
/ 252\end{array}$ & $\begin{array}{l}++ \\
++ \\
++ \\
+\end{array}$ & $\begin{array}{l}\text { No gain in tolerance with increase in carbo- } \\
\text { hydrate; proportional doses of insulin required }\end{array}$ \\
\hline
\end{tabular}


TABLE VI-Continued

\begin{tabular}{|c|c|c|c|c|c|c|c|c|c|c|}
\hline 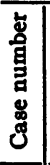 & 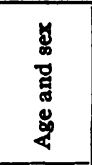 & 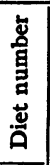 & 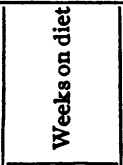 & 量 & 崫 & 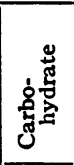 & 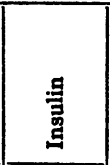 & 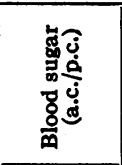 & 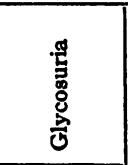 & Remarks \\
\hline 6 & $\begin{array}{l}\text { years } \\
44 \mathrm{~F}\end{array}$ & $\begin{array}{l}1 \\
2 \\
3 \\
4 \\
5 \\
6 \\
7\end{array}$ & \begin{tabular}{|cc|c|} 
& & \\
& 4 \\
& 8 \\
$1 \frac{1}{2}$ & years \\
& 8 \\
& 8 \\
3 & 3 \\
& 2
\end{tabular} & $\begin{array}{c}\text { grams } \\
60 \\
60 \\
70 \\
60 \\
60 \\
60 \\
60\end{array}$ & $\begin{array}{c}\text { grams } \\
125 \\
125 \\
135 \\
135 \\
140 \\
140 \\
125\end{array}$ & $\begin{array}{c}\text { grams } \\
150 \\
150 \\
130 \\
130 \\
180 \\
200 \\
150\end{array}$ & \begin{tabular}{|c|} 
units per \\
24 hours \\
22 \\
32 \\
$32-$ \\
39 \\
32 \\
40 \\
40 \\
32
\end{tabular} & $\begin{array}{l}\text { mgm. per } \\
10 j \text { cc. }\end{array}$ & $\begin{array}{c}++ \\
\\
++++ \\
++++\end{array}$ & $\begin{array}{l}\text { Marked glycosuria and acetonuria with high } \\
\text { carbohydrate. Diet } 7 \text { same as Diet } 2 \text { with } \\
\text { practically same insulin requirement. No } \\
\text { tendency to gain tolerance }\end{array}$ \\
\hline 7 & $53 \mathrm{~F}$ & $\begin{array}{l}1 \\
2 \\
3\end{array}$ & $\begin{array}{l}4 \\
4 \\
4\end{array}$ & $\begin{array}{l}100 \\
100 \\
100\end{array}$ & $\begin{array}{l}179 \\
134 \\
114\end{array}$ & $\begin{array}{l}200 \\
250 \\
350\end{array}$ & $\begin{array}{l}\mathbf{0} \\
\mathbf{0} \\
\mathbf{0}\end{array}$ & $\begin{array}{r}142 / 205 \\
/ 160 \\
/ 212\end{array}$ & $\begin{array}{l}+ \\
+\end{array}$ & $\begin{array}{l}\text { Gaining tolerance with high carbohydrate, } \\
\text { glycosuria and blood sugar practically un- } \\
\text { changed on much higher carbohydrate }\end{array}$ \\
\hline 8 & $33 \mathrm{M}$ & $\begin{array}{l}1 \\
2 \\
3\end{array}$ & $\begin{array}{c}2 \text { years } \\
10 \\
4\end{array}$ & $\begin{array}{l}70 \\
57 \\
57\end{array}$ & $\begin{array}{r}150 \\
97 \\
97\end{array}$ & $\begin{array}{l}125 \\
114 \\
150\end{array}$ & $\begin{array}{l}30 \\
45 \\
52\end{array}$ & $\begin{array}{r}138 / 246 \\
157 / 173 \\
/ 194\end{array}$ & $\begin{array}{l}++ \\
+\end{array}$ & $\begin{array}{l}\text { Carbohydrate decreased, insulin increased be- } \\
\text { cause of glycosuria } \\
\text { Higher carbohydrate requires proportional in- } \\
\text { crease in insulin }\end{array}$ \\
\hline 9 & $56 \mathrm{~F}$ & $\begin{array}{l}1 \\
2 \\
3 \\
4 \\
5\end{array}$ & $\begin{array}{l}\mathbf{4} \\
\mathbf{4} \\
\mathbf{4} \\
\mathbf{4} \\
\mathbf{4}\end{array}$ & $\begin{array}{l}57 \\
60 \\
60 \\
60 \\
60\end{array}$ & $\begin{array}{r}98 \\
90 \\
100 \\
100 \\
100\end{array}$ & $\begin{array}{l}114 \\
268 \\
240 \\
300 \\
300\end{array}$ & $\begin{array}{l}25 \\
35 \\
35 \\
35 \\
30\end{array}$ & $\begin{array}{r}161 / 130 \\
/ 145 \\
/ 148 \\
/ 152\end{array}$ & & $\begin{array}{l}\text { Enormous increase in carbohydrate permitted, } \\
\text { with practically no increase in insulin required }\end{array}$ \\
\hline 11 & $43 \mathrm{~F}$ & $\begin{array}{l}1 \\
2 \\
3 \\
4 \\
5\end{array}$ & $\begin{array}{c}8 \\
3 \\
16 \\
1 \text { year } \\
4\end{array}$ & $\begin{array}{l}60 \\
60 \\
60 \\
60 \\
60\end{array}$ & $\begin{array}{r}100 \\
125 \\
125 \\
60 \\
50\end{array}$ & $\begin{array}{r}80 \\
150 \\
150 \\
200 \\
250\end{array}$ & $\begin{array}{l}10 \\
50 \\
30 \\
30 \\
30\end{array}$ & $\begin{array}{l}/ 105 \\
/ 154 \\
/ 168 \\
/ 180\end{array}$ & & $\begin{array}{l}\text { Reduction of insulin with higher carbohydrate } \\
\text { Only slight rise in blood sugar and occasional } \\
\text { glycosuria with very large increase in carbo- } \\
\text { hydrate }\end{array}$ \\
\hline 13 & $33 \mathrm{~F}$ & $\begin{array}{l}1 \\
2 \\
3 \\
4 \\
5 \\
6\end{array}$ & $\begin{array}{r}1 \\
2 \\
8 \\
4 \\
4 \\
20\end{array}$ & $\begin{array}{l}60 \\
60 \\
60 \\
60 \\
60 \\
60\end{array}$ & $\begin{array}{r}125 \\
125 \\
150 \\
100 \\
80 \\
60\end{array}$ & $\begin{array}{l}150 \\
250 \\
150 \\
170 \\
210 \\
300\end{array}$ & $\begin{array}{r}0 \\
45 \\
30 \\
20 \\
0 \\
0\end{array}$ & $\begin{array}{c}146 / 206 \\
/ 80 \\
/ 96 \\
\\
/ 112 \\
/ 126\end{array}$ & ++ & $\begin{array}{l}\text { Gain in tolerance with high carbohydrate until } \\
\text { insulin could be omitted entirely }\end{array}$ \\
\hline 14 & $53 \mathrm{~F}$ & $\begin{array}{l}\mathbf{1} \\
\mathbf{2} \\
\mathbf{3} \\
\mathbf{4} \\
\mathbf{5} \\
\end{array}$ & $\begin{array}{l}8 \\
2 \\
2 \\
2 \\
2\end{array}$ & $\begin{array}{r}100 \\
100 \\
70 \\
70 \\
70\end{array}$ & $\begin{array}{r}170 \\
170 \\
60 \\
60 \\
60\end{array}$ & $\begin{array}{l}200 \\
200 \\
225 \\
225 \\
225\end{array}$ & $\begin{array}{l}55 \\
40 \\
40 \\
35 \\
30\end{array}$ & $\begin{array}{r}206 / 110 \\
1 / 115 \\
184 / / 105 \\
/ 140\end{array}$ & & $\begin{array}{l}\text { Gain in tolerance on high carbohydrate; reduc- } \\
\text { tion in insulin }\end{array}$ \\
\hline 15 & $55 \mathrm{~F}$ & $\begin{array}{l}1 \\
2 \\
3 \\
4 \\
5 \\
6 \\
7\end{array}$ & $\begin{array}{l}6 \\
2 \\
2 \\
2 \\
6 \\
4 \\
2 \\
2\end{array}$ & $\begin{array}{l}70 \\
70 \\
70 \\
70 \\
70 \\
70 \\
70\end{array}$ & $\begin{array}{r}119 \\
89 \\
70 \\
70 \\
70 \\
70 \\
70\end{array}$ & $\begin{array}{l}140 \\
210 \\
250 \\
250 \\
250 \\
250 \\
250\end{array}$ & $\begin{array}{l}70 \\
65 \\
65 \\
55 \\
40 \\
30 \\
15\end{array}$ & $\begin{array}{r}175 / 166 \\
/ 140 \\
/ \\
/ 130 \\
/ 144 \\
/ 156 \\
/ 149\end{array}$ & & $\begin{array}{l}\text { Gain in tolerance as carbohydrate raised; re- } \\
\text { duction in insulin }\end{array}$ \\
\hline
\end{tabular}

accompanied in many cases by decreased sugar tolerance or frank diabetes. Thyroidectomy may diminish the severity of the diabetes and increase the effectiveness of insulin in such patients (27).

Claude Bernard's piqûre directed attention to the importance of the nervous system in carbohydrate metabolism. Injury to or tumors affect- ing the hypothalamus may cause diabetes. Davis (28) has been able to produce lesions in the hypothalamus which greatly diminish the severity of the diabetes following pancreatectomy. Animals in which the adrenal medullary tissue has been removed, or the adrenal sympathetic nerve supply has been severed, gain in sugar tolerance 
and are hypersensitive to insulin (29). De Takats and Fenn have shown that splanchnic nerve section may increase the effectiveness of insulin in and ameliorate human diabetes (30).

It seems evident that all of the factors mentioned must operate through the liver since it serves as the source of the blood sugar during fasting. Diabetics with various hepatic disorders may require disproportionately large doses of insulin (31). Himsworth (32) concludes from a series of interesting experiments that carbohydrate ingestion increases susceptibility to insulin by causing an increase in an hypothetical insulin activator produced in the liver. The evidence supporting the existence of such a factor is as yet inconclusive.

Present clinical and experimental knowledge indicates that many cases of diabetes may not be due primarily to inadequate production of insulin. The central nervous system, the pituitary, the thyroid, the suprarenals and the liver form a chain of factors influencing the blood sugar level and the storage and combustion of carbohydrate. Extra-pancreatic factors may, by interfering with the action of endogenous insulin, be of importance in the etiology of diabetes.

\section{SUMMARY AND CONCLUSIONS}

1. The history of the dietary management of diabetes reveals that the greatest students of the disease have differed widely concerning the optimum balance of the various foodstuffs. The fact that many diabetics will gain tolerance on high carbohydrate diets has been reemphasized by recent workers and has attracted much attention. Less attention has been paid to the equally important fact that other diabetics experience deleterious results when allowed large amounts of carbohydrate.

2. A group of diabetics intensively studied over a three year period fall into two classes, the relatively insulin-sensitive and the relatively insulinresistant. The resistant type tend to be older, frequently are obese, often have hypertension, and are less subject to acidosis and coma. The sensitive type are usually younger, thin, or of normal nutrition, have low blood pressures and a marked tendency to acidosis. The two groups cannot be separated according to severity, since if the insulin requirement be used as the criterion, the resistant group would seem the more severe, but judged by the tendency to acidosis, the sensitive group would seem to have the more serious type of disease.

3. The insulin-sensitive group failed to gain tolerance on high carbohydrate diets. Only one exception among eight patients was noted to this general rule. Relatively high fat was well borne.

4. The relatively resistant group without exception gained tolerance upon a high carbohydrate intake. In several instances this was shown to be accompanied by increased sensitivity to insulin.

5. Recent studies have shown the probable importance of extrapancreatic influences upon carbohydrate metabolism. It is interesting to note that our insulin-sensitive patients resemble in many respects the partially pancreatectomized animal. They respond well to exogenous insulin, but seem to produce too little of the endogenous hormone. When subjected to the excessive burden of a high carbohydrate intake they may lose tolerance, perhaps as the result of overburdening the damaged or numerically decreased pancreatic islets. Relatively resistant patients, however, react as if the endogenous insulin supply were adequate in amount, but operating under the handicap of inhibiting factors.

6. Studies such as those here described may prove useful in indicating the type of diet which will lead to maximum individual carbohydrate tolerance. The evidence at present indicates that the insulin-resistant type may be expected to gain tolerance with high carbohydrate, while the insulin-sensitive type may either fail to gain or may lose tolerance with excessive carbohydrate ingestion.

\section{BIBLIOGRAPHY}

1. Woodyatt, R. T., Dietary trends (Round Table Conference on diabetes mellitus). Bull., New York Acad. Med., 1934, 10, 335.

von Noorden, C. H., Die Zuckerkrankheit und ihre Behandlung. Hirschwald, Berlin, 1910, 5th ed.

Falta, W., Die Amylazeen (Mehlfruchte) in der Kost der Zuckerkranken. Wien. klin. Wchnschr., 1918. 31, 1199.

2. Naunyn, Bernard, Der Diabetes Melitus. Hölder, Wien., 1906, 2d ed.

3. Allen, F. M., Stillman, Edgar, and Fitz, Reginald, Total dietary regulation in the treatment of diabetes. Monograph No. 11, Rockefeller Inst., New York, 1919. 
4. Newburgh, L. H., and Marsh, P. L., The use of a high fat diet in the treatment of diabetes mellitus. Arch. Int. Med., 1920, 26, 647.

Newburgh, L. H., and Waller, D. S., Studies of diabetes mellitus. Evidence that the disability is concerned solely with the metabolism of glucose. The mode of action of insulin. J. Clin. Invest., 1932, 11, 995.

5. Petrén, K., Ueber die Gründe der diätetischen Behandlung des Diabetes, besonders des Diabetes gravis. München. med. Wchnschr., 1927, 74, 1123.

6. Rosenberg, M., Beitrag zur kohlenhydratreichen, fettarmen Diät bei Diabetes. Deutsche med. Wchnschr., 1933, 59, 320.

7. Sansum, W. D., Blatherwick, N. R., and Bowden, $R$., The use of high carbohydrate diets in the treatment of diabetes mellitus. J. A. M. A., 1926, 86, 178.

Gray, P. A., and Sansum, W. D., Higher carbohydrate diet method in diabetes mellitus. An analysis of 1,005 cases. J. A. M. A., 1933, 100, 1580.

Adlersberg, D., and Porges, O., Zur Theorie und Praxis der Kurativen Diabetesbehandlung. Klin. Wchnschr., 1926, 5, 1451.

Geyelin, H. R., Recent studies on diabetes in children. Atlantic Med. J., 1926, 29, 825.

The treatment of diabetes with insulin (after ten years), contrasting the effects of normal and of the older diabetic diets. J. A. M. A., 1935, 104, 1203.

Rabinowitch, I. M., Experiences with high carbohydrate, low calorie diet for the treatment of diabetes mellitus. Canad. M. A. J., 1930, 23, 489.

8. Joslin, E. P., The Treatment of Diabetes Mellitus. Lea \& Febiger, Philadelphia, 1928, 4th ed.

9. MacBryde, C. M., Insulin resistance in diabetes mellitus. Arch. Int. Med., 1933, 52, 932.

10. Falta, W., and Boller, R., Insulärer und insulinresistenter Diabetes. Klin. Wchnschr., 1931, 10, 438.

11. Somogyi, M., Notes on sugar determination. J. Biol. Chem., 1926, 70, 599.

12. Shaffer, P. A., and Hartmann, A. F., The iodometric determination of copper and its use in sugar analysis. II. Methods for the determination of reducing sugars in blood, urine, milk and other solutions. J. Biol. Chem., 1921, 45, 365.

13. Hamman, L., and Hirschman, I. I., Effects upon the blood sugar of the repeated ingestion of glucose. Bull. Johns Hopkins Hosp., 1919, 30, 306.

14. Staub, H., Bahnung im intermediären Zuckerstoffwechsel. Biochem. Ztschr., 1921, 118, 93.

15. Traugott, K., Uber das Verhalten des Blutzuckerspiegels bei wiederholter und verschiedener Art enteraler Zuckerzufuhr und dessen Bedeutung für die Leberfunktion. Klin. Wchnschr., 1922, 1, 892.

16. Foster, G. L., Studies on carbohydrate metabolism. II. An interpretation of the blood sugar phenomena following the ingestion of glucose. J. Biol. Chem., 1923, 55, 303.
17. du Vigneaud, V., and Karr, W. G., Carbohydrate utilization. I. Rate of disappearance of $d$-glucose from the blood. J. Biol. Chem., 1925, 66, 281.

18. Lennox, W. G., Stimulation of the sugar-regulating mechanism as shown by duplicate blood sugar curves. J. Biol. Chem., 1927, 73, 237.

19. Sweeney, J. S., Dietary factors that influence the dextrose tolerance test. A preliminary study. Arch. Int. Med., 1927, 40, 818.

20. Macleod, J. J. R., Diabetes as a physiological problem. Lancet, 1930, 2, 512.

21. Abderhalden, E., and Wertheimer, E., Studien über den Einfluss der Ernährung auf die Wirkung bestimmter Inkretstoffe. III. Insulin- und Adrenalinwirkung bei Verabreichung "Sauer" bezw. "basischer" Nahrung. Arch. f. d. ges. Physiol., 1924, 205, 559.

22. Bainbridge, $H$. W., The reduced sensitivity to insulin of rats and mice fed on a carbohydrate-free excess-fat diet. J. Physiol., 1925, 60, 293.

23. Hynd, A., and Rotter, D. L., Studies on the metabolism of animals on a carbohydrate-free diet. Variations in the sensitivity towards insulin of different species of animals on carbohydrate-free diets. Biochem. J., 1931, 25, 457.

24. Watson, E. M., and Wharton, M. A., A comparison of various diets in the treatment of diabetes mellitus. Quart. J. Med., 1935, 4, 277.

25. Warren, S., The pathology of the pancreas in nondiabetic persons. A study of 156 consecutive autopsies on nondiabetic patients. Arch. Int. Med., 1929, 44, 663.

26. Cushing, H., and Davidoff, L. M., The pathological findings in four autopsied cases of acromegaly with a discussion of their significance. Monograph No. 22, Rockefeller Inst., New York, 1927.

Cushing, $H$., The basophil adenomas of the pituitary body and their clinical manifestations (pituitary basophilism). Bull. Johns Hopkins Hosp., 1932, 50, 137.

Lucke, H., Hypophysäre Magersucht und Insulin. Klin. Wchnschr., 1932, 11, 1988.

Houssay, B. A., and Biasotti, A., The hypophysis, carbohydrate metabolism and diabetes. Endocrinology, 1931, 15, 511.

Lucke, H., Heydemann, E. R., and Duensing, F., Untersuchungen über den Wirkungs-Mechanismus des kontrainsulären Hormons des Hypophysenvorderlappens. I. Hypophysenvorderlappen, Schilddrüse und Kohlehydratstoff wechsel. Ztschr. f. d. ges. exper. Med., 1933, 91, 106.

Lucke, H., Heydemann, E. R., and Hahndel, H., II. Hypophysenvorderlappen, Nebenniereninsuffizienz und Kohlehydratstoff wechsel. Idem, p. 483.

III. Hypophysenvorderlappen, Nebennierenentnervung und Kohlehydratstoff wechsel.

Lucke, H., and Hahndel, H., VI. Die Möglichkeit eines biologischen Nachweises des kontrainsulären Hormons im Liquor cerebrospinalis. Idem, p. 704. 
Viale, G., Die Bedeutung der Nebennierenrinde für den Stoffwechsel der Kohlehydrate. Klin. Wchnschr., 1933, 12, 467.

Long, C. N. H., and Lukens, F. D. W., Observations on adrenalectomized, depancreatized animals. J. Clin. Invest. (Proc.), 1934, 13, 685.

Long, C. N. H., Recent advances in carbohydrate metabolism with particular reference to diabetes mellitus. Ann. Int. Med., 1935, 9, 166.

Walters, W., Wilder, R. M., and Kepler, E. T., The suprarenal cortical syndrome with presentation of ten cases. Ann. Surg., 1934, 100, 670.

27. Strauss, H., Uber insulin-resistente Diabetiker. Klin. Wchnschr., 1925, 4, 491.

Kogan, V., Antagonismus und Korrelation zwischen Pankreas, Nebennieren und Hypophysis. Die Erkrankungen des Pankreas. Ztschr. f. klin. Med., 1926, 104, 457.

Serio, F., Zur Kenntnis der insulinresistenten Diabetes. Klin. Wchnschr., 1931, 10, 1998.

28. Davis, L., The relation of the hypophysis, hypothalamus and the autonomic nervous system to carbohydrate metabolism. Ann. Surg., 1934, 100, 654.

29. Rupp, F., Uber den Einfluss des Nervensystems auf den Zuckergehalt des Blutes. Ztschr. f. d. ges. exper. Med., 1924-1925, 44, 476.

Ciminata, A., Einfluss der Durchschneidung der Nebennieren-nerven auf den Diabetes Mellitus. Klin. Wchnschr., 1932, 11, 774.

Barnes, B. O., Scott, V. B., Ferrill, H. W., and
Rogoff, J. M., Effects of partial adrenalectomy on experimental diabetes and on sensitivity to insulin. Proc. Soc. Exper. Biol. and Med., 1934, 31, 524.

Barnes, B. O., Dix, A. S., and Rogoff, J. M., Effect of adrenalin on insulin sensitivity of partially adrenalectomized and of hypophysectomized dogs. Proc. Soc. Exper. Biol. and Med., 1934, 31, 1145.

de Takats, G., and Cuthbert, F. P., The effect of coeliac ganglionectomy on the sugar tolerance of dogs. Am. J. Physiol., 1932, $102,614$.

30. de Takats, G., and Fenn, G. K., Bilateral splanchnic nerve section in a juvenile diabetic. Ann. Int. Med., 1933, 7, 422.

31. Leech, E. B., Bronzed diabetes: Its reactions to dietetic and insulin treatment. Lancet, 1923, 2, 69.

Widal, F., Abrami, P., Weill, A., and Laudat, Action dissocieé de l'insuline sur la glycosurie et l'acétonurie. Presse méd., 1924, 32, 253.

Chabrol, E., and Hébert, P., Paris med., 1925, 1, 453.

Boller, R., and Uberrack, K., Die Insulintoleranz bei Fällen von Ikterus. Klin. Wchnschr., 1932, 11, 671.

32. Himsworth, H. P., The physiological activation of insulin. Clin. Science, 1933, 1, 1.

Dietetic factors influencing glucose tolerance and activity of insulin. J. Physiol., 1934, 81, 29.

High carbohydrate diets and insulin efficiency. Brit. M. J., 1934, 2, 57. 